Direct-write non-linear photolithography for semiconductor nanowire characterization

This article has been downloaded from IOPscience. Please scroll down to see the full text article.

2012 Nanotechnology 23335704

(http://iopscience.iop.org/0957-4484/23/33/335704)

View the table of contents for this issue, or go to the journal homepage for more

Download details:

IP Address: 130.56.107.38

The article was downloaded on 05/10/2012 at 10:03

Please note that terms and conditions apply. 


\title{
Direct-write non-linear photolithography for semiconductor nanowire characterization
}

\author{
P Parkinson, N Jiang, Q Gao, H H Tan and C Jagadish \\ Department of Electronic Materials Engineering, Research School of Physics and Engineering, \\ The Australian National University, Canberra, ACT 0200, Australia \\ E-mail: patrick.parkinson@anu.edu.au
}

Received 12 March 2012, in final form 12 July 2012

Published 3 August 2012

Online at stacks.iop.org/Nano/23/335704

\begin{abstract}
A practical bottleneck prohibiting the rapid, confident and damage-free electrical contacting of vapour-liquid-solid grown nanowires arises from the random spatial distribution and variation in quality of the nanowires, and the contact dimensions required. Established techniques such as electron-beam lithography or focused ion-beam deposition have challenges in scaling, damage or complexity that can make a large statistical sample difficult. We present a direct laser-writing technique to allow rapid electrical contacting of nanowires on a large variety of substrates.
\end{abstract}

S Online supplementary data available from stacks.iop.org/Nano/23/335704/mmedia

(Some figures may appear in colour only in the online journal)

\section{Introduction}

The intense development in the field of semiconductor nanowires over the past decade has presented many opportunities to exploit new physics arising from nanoscale materials, particularly in the field of optoelectronics [1, 2]. However, a major obstacle limiting the rapid electrical characterization of such nanowires is the bottleneck associated with electrical contacting. Current techniques such as electron-beam lithography (EBL) [3], focused ion-beam (or electron-beam induced) deposition (FIB) [4], and advanced optical techniques such as time-resolved terahertz spectroscopy (THz) [5] are all able to characterize nanowires; however, each has limitations, such as the substrate used (somewhat conducting for EBL and FIB, and insulating for $\mathrm{THz}$ spectroscopy), the time required to successfully contact a suitable number of wires to gain a statistically accurate insight into the nanowire properties (EBL and FIB), or conversely a lack of the sensitivity required to study the nanowires on a wire-by-wire basis $(\mathrm{THz})$.

The challenge is related to that of precisely locating single nanowires; in particular, nanowires grown using the vapour-liquid-solid mechanism are typically randomly distributed on the growth substrate. Techniques such as aligned rubbing [6], patterned-substrate growth [7] or even nano-manipulator based positioning $[8,9]$ have been developed, but their lack of extensibility to either large (patterned substrate and nano-manipulator) or small scale (aligned rubbing) have limited their application.

In this paper, we describe a non-linear laser photolithography technique [10] that is quick, flexible, and possesses a high enough spatial resolution to contact single nanowire samples on arbitrary substrates. In addition, this optical based technique can avoid any potential contamination associated with extended ion-beam or electron-beam irradiation. Previously, linear (single photon) direct laser writing has been used to define contacts on nanomaterials such as graphene [11], to positive effect. The primary advantages of the present technique are the easy visibility of the nanowires through the photoresist (under UV-free light) and the possibility of using lower-power laser illumination to perform photoluminescence (PL) measurements in situ, together allowing direct writing of contact patterns during the first processing run. This removes the need for high precision alignment markers, and the vacuum-free nature of optical exposure significantly increases the throughput of this technique in comparison with EBL. Coupled with the ability to make electrical contacts 


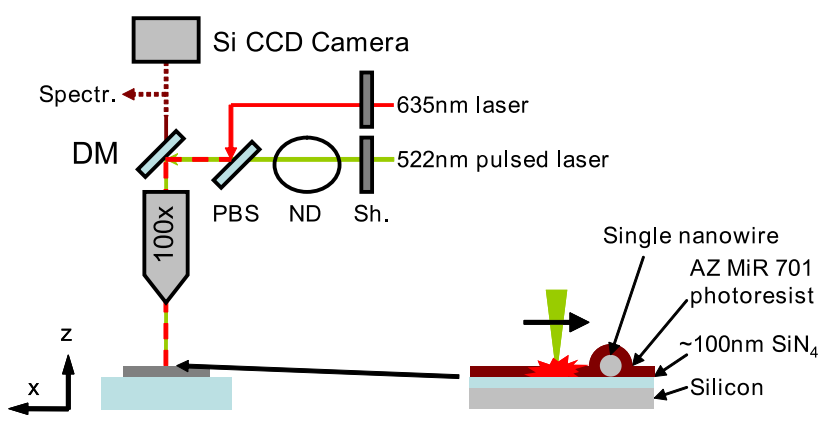

Figure 1. Schematic of the working principle for direct laser writing. DM: dichroic mirror, PBS: partial beam splitter (50:50), ND: computer controlled variable neutral density filter, $\mathrm{Sh}$ : computer controlled shutter, Spectr: grating spectrometer.

on arbitrary substrates (insulators such as thick oxide layers, glass and flexible transparent substrates such as PDMS provide interesting opportunities to exploit) and a lack of ion or energetic electron-beam irradiation, this technique possesses significant possibilities.

\section{Experimental details}

The schematic in figure 1 depicts the layout of the optical system. For photoluminescence measurements, a $635 \mathrm{~nm}$ solid-state continuous-wave laser was used, providing $2.5 \mathrm{~mW}$ at the focal spot. The photoluminescence was passed through an $800 \mathrm{~nm}$ long pass filter, directed onto a monochromator with a 750 grooves $\mathrm{mm}^{-1}$ grating and detected using a cooled CCD (for spectral measurements) or an avalanche photodiode (for mapping). For direct writing, a pulsed laser operating at $1044 \mathrm{~nm}$ and frequency doubled to $522 \mathrm{~nm}$ with a pulse duration of $\approx 300 \mathrm{fs}$ and a repetition rate of $20 \mathrm{MHz}$ was used. By imaging via a microscope equipped with an extra-long working distance $100 \times$ lens, a spot with a diameter of approximately $2.5 \mu \mathrm{m}$ was formed on a substrate. The laser-writing power was varied by two orders of magnitude from $I_{0} / 100$ to $I_{0}=2.8 \mathrm{~mW}$ (approximately $60 \mathrm{~kW} \mathrm{~cm}{ }^{-2}$ ) using a computer controlled variable neutral density filter. The laser spot was scanned across the substrate by use of a two-axis $X Y$ motorized translation stage, capable of moving up to $250 \mu \mathrm{m}$ per second with a maximum range of $25 \mathrm{~mm} \times$ $6 \mathrm{~mm}$. Both lasers could be blocked using computer controlled shutters with $\approx 15 \mathrm{~ms}$ switching times.

$\mathrm{GaAs}-\mathrm{AlGaAs}$ core-shell nanowires were grown using an established Au catalysed MOCVD process, with a core diameter of $50 \mathrm{~nm}$, and a length of $\sim 7 \mu \mathrm{m}$ following a two-temperature growth process similar to that previously published [12], and were removed from the growth substrate by ultrasonication in iso-propyl-alcohol for $5 \mathrm{~s}$. These nanowires were of particularly high quality and had significant room-temperature luminescence [13]. A substrate consisting of a silicon wafer coated with approximately $100 \mathrm{~nm}$ of silicon nitride was prepared using PECVD at $300^{\circ} \mathrm{C}$. The nanowires were dispersed onto the new substrate by drop casting and subsequent drying at room temperature. The positive photoresist AZ MiR 701 was spin coated over

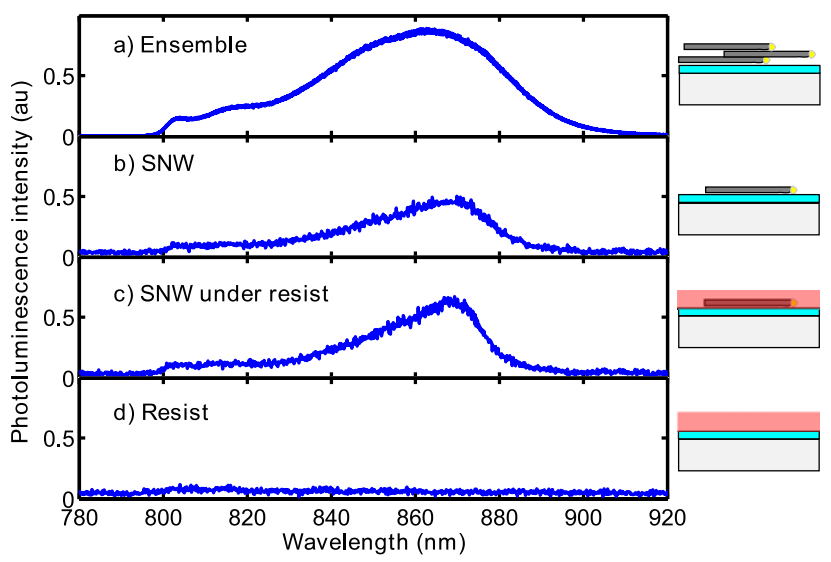

Figure 2. The room-temperature photoluminescence spectra for (a) a nanowire ensemble and (b) a single nanowire. After covering with the photoresist, the photoluminescence is shown for (c) a single nanowire and (d) the resist. All spectra are shown on the same intensity scale, except for the ensemble (on 1/10 scale).

the nanowires at $6000 \mathrm{rpm}$ for $120 \mathrm{~s}$ to provide a thin film (approximately $0.75 \mu \mathrm{m}$ as measured by step profilometer), and the resist was soft baked for $60 \mathrm{~s}$ at $85^{\circ} \mathrm{C}$. After laser exposure, the sample was hard baked at $110^{\circ} \mathrm{C}$ for $60 \mathrm{~s}$, followed by developing using AZ $326 \mathrm{MiF}$ developer for $60 \mathrm{~s}$ at room temperature. The sample was metallized using e-beam evaporation of Ti:Au with thicknesses of $10 \mathrm{~nm}$ and $200 \mathrm{~nm}$ respectively before lift-off in acetone at room temperature.

\section{Results}

Initially, photoluminescence measurements were carried out at room temperature for the nanowires transferred to a silicon substrate, as shown in figures 2(a) and (b). The luminescence can clearly be seen, with a peak at $860-870 \mathrm{~nm}$ corresponding to emission from the GaAs core. After coating with the photoresist, luminescence was still visible under the same excitation conditions, with a similar signal size (figure 2(c)). Under the illumination conditions described $(2.5 \mathrm{~mW}, 635 \mathrm{~nm}$, spot size $2.5 \mu \mathrm{m})$ no exposure of the resist was observed, even with extended measurement times of several seconds. The photoluminescence allows the separation of microscopically observed features into high-quality nanowires with room-temperature signal, and non-emissive contamination such as dust or low-quality nanowires. Figure 3 shows a combined optical and photoluminescence mapping scan-it can be seen that a feature (on the upper right of figure 3(c)) that might be mistaken for a single nanowire can be eliminated by the lack of photoluminescence signal, while the nanowire itself can be clearly seen. In addition, in more complex nanowire structures such as axial heterostructures, it is anticipated that this technique may provide information on which end of the nanowire requires contacting and, further, allow the discrimination of multiple species of nanowire (i.e. indium phosphide, gallium arsenide, etc) on a single substrate.

To optimize the direct-write process, a series of test lines were exposed at a range of write speeds, from 5 to 
a) Visible image

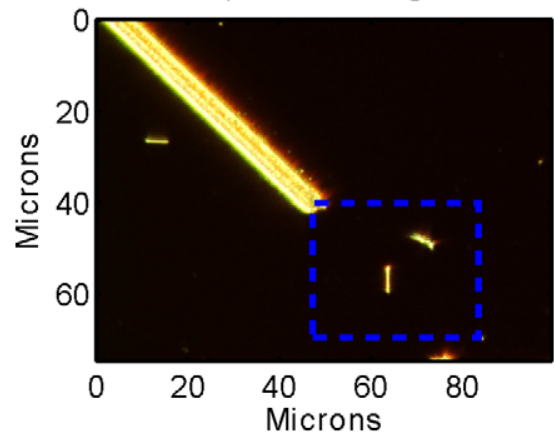

c) Combined image

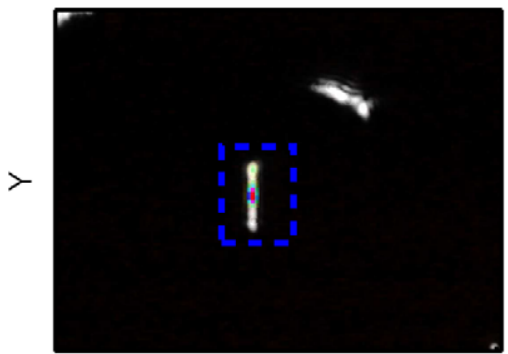

$X$ b) PL map

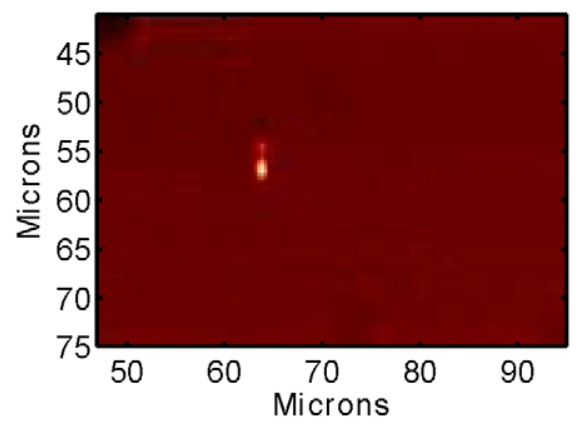

d) Magnified image

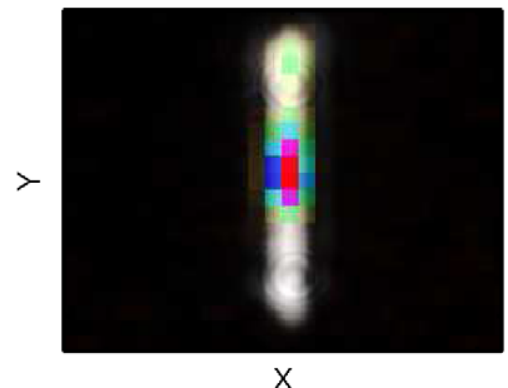

Figure 3. (a) An optical micrograph of a single nanowire. (b) A photoluminescence map covering the area marked with the blue square in (a). The large feature to the upper left is an alignment mark on the substrate. (c) A scaled, overlayed combined image with photoluminescence intensity shown in colour, and (d) a magnified section surrounding the nanowire (indicated by the blue square in (c)) are shown below.

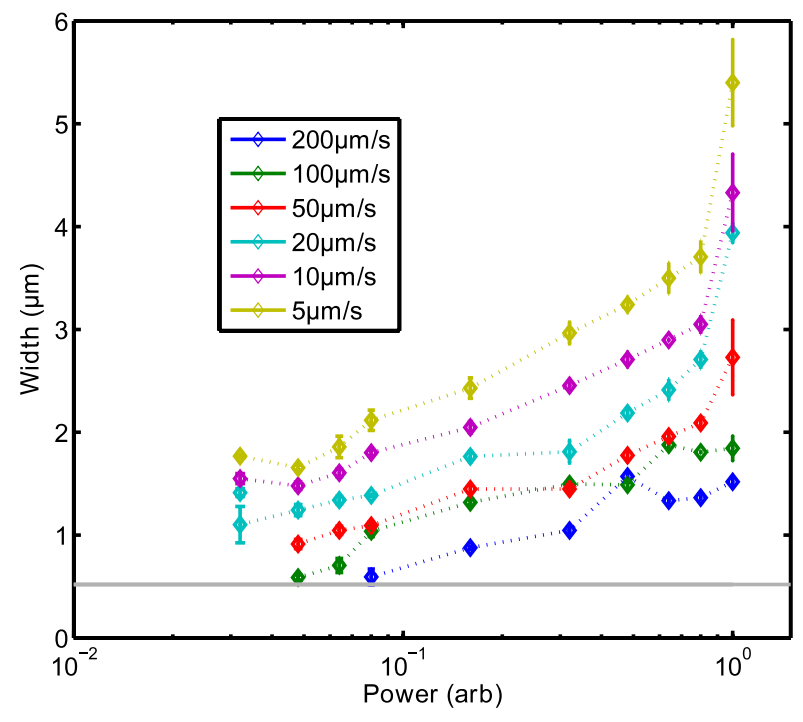

Figure 4. The width of written lines as a function of laser power and write speed. The error bars represent the standard error in three measurements of the same line at different points. The solid line represents the wavelength of the laser used, a rough guide to the diffraction-limited result.

$200 \mu \mathrm{m} \mathrm{s}^{-1}$, and over a range of powers, from $I_{0} / 100$ to $I_{0}$ (see supplementary material I available at stacks.iop. org/Nano/23/335704/mmedia for optical micrographs of the written calibration lines). After exposure and developing, the written line widths were measured using a calibrated optical microscope as shown in figure 4 . It was found that line widths from 0.6 to $6 \mu \mathrm{m}$ could be reliably fabricated, providing the resolution and speed to accurately contact single nanowires. Moreover, by using computer control of the laser power, it becomes possible to use low-power, low-speed writing to give narrow lines widths for contacting, followed by high-power, high-speed writing to create larger features such as contact pads. With a combination of such fast and slow written elements, writing of contact pads for a single nanowire in less that $5 \mathrm{~min}$ was feasible, and much faster may be possible with carefully optimized and designed equipment. The approximate dose was calculated for the range of laser fluence and write speeds measured. This is given by

$$
D=P^{\alpha} / S,
$$

where $P$ is the laser power (in arbitrary units), $S$ is the write speed-equivalent to the inverse dwell time-and $\alpha$ is either 1 or 2 for a one-photon (linear) or two-photon (non-linear) exposure process, respectively. By comparison of the calculated dose with the visibility of the written lines, the critical dose can be calculated. It was found that a critical dose below which the lines were not fully written could be found for $\alpha=2$, but not for the $\alpha=$ 1 (see supplementary material II available at stacks.iop. org/Nano/23/335704/mmedia for more details and tables of this calculation). Two primary benefits result from the non-linear process underlying photoresist exposure: the possibility of using high-power continuous-wave laser light for photoluminescence measurement without resist exposure, and that of sub-wavelength feature definition [14]. To achieve the highest possible spatial resolution, additional steps must 

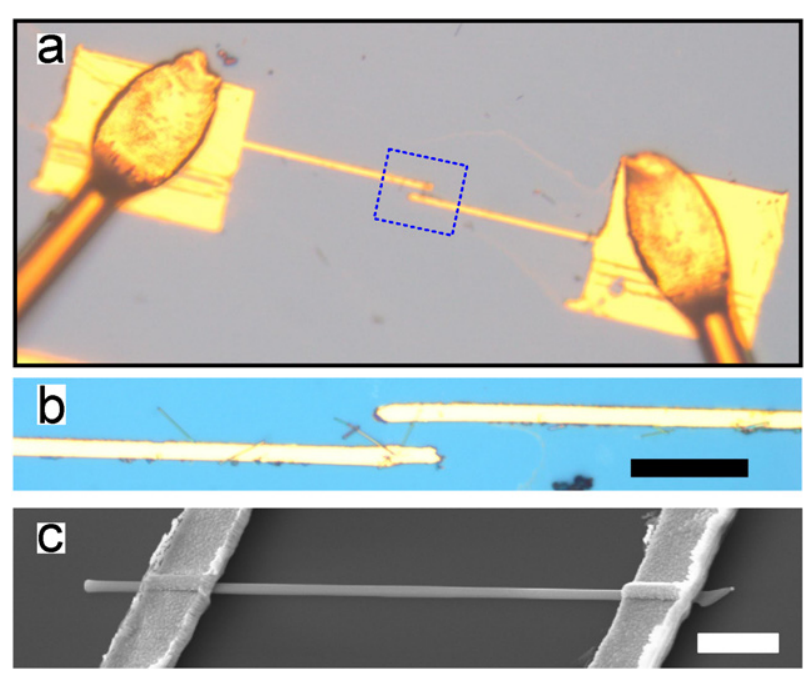

Figure 5. (a), (b) An optical microscope image of a contacted GaAs nanowire after metallization and wire bonding. The blue square indicates the region shown in the high magnification centre image (b). The scale bar is $20 \mu \mathrm{m}$. (c) A scanning electron micrograph of a similar contacted nanowire showing micron-scale contact pads. The scale bar is $1 \mu \mathrm{m}$.

be taken. Specifically, the thickness of the photoresist should be minimized to prevent incomplete exposure and associated 'closed' lines, and the $z$-axis (focus) should be carefully controlled. While such improvements are of significant interest for future optimization of the technique, the sub-micron resolution currently achieved provides ample opportunity for implementation of this technique.
A direct-write contacted nanowire is shown in figure 5 after metallization and wire bonding. As can be seen, it is straightforward to selectively contact a single nanowire with micron-scale resolution and line width. Subsequent electrical characterization was carried out to determine the quality of the contacts and nanowire. Figure 6 shows the dark and light (under illumination with the pulsed $522 \mathrm{~nm}$ laser) current-voltage (IV) measurements. Several features are noticeable. Firstly, the dark current at room temperature is low (with a differential resistance of over $5 \mathrm{~T} \Omega$ at $3 \mathrm{~V}$ ). Both dark and light current exhibit an asymmetric Schottky-like profile, most likely due to the effect of any native oxide and the unremoved AlGaAs shell. However, the nanoamp level of the light current is indicative of a well functioning device [15]. No effort was taken to make specifically Ohmic contacts, for instance oxide removal with an acid dip, surface passivation with ammonium sulfide [16] or rapid thermal annealing of the contacts.

\section{Conclusion}

With an appropriate choice of photoresist thickness and laser power, along with more careful focusing (using a high numerical aperture lens) and carefully controlled write speeds, it is anticipated that a reduction in feature size may be realized. Indeed, by exploiting the non-linear nature of the exposure process at $522 \mathrm{~nm}$, sub-wavelength features may be exposed without resorting to costly and time-consuming electron-beam lithography. By removing the bottleneck in the electrical contacting of good-quality nanowires, and permitting in situ nanowire screening to be carried out using
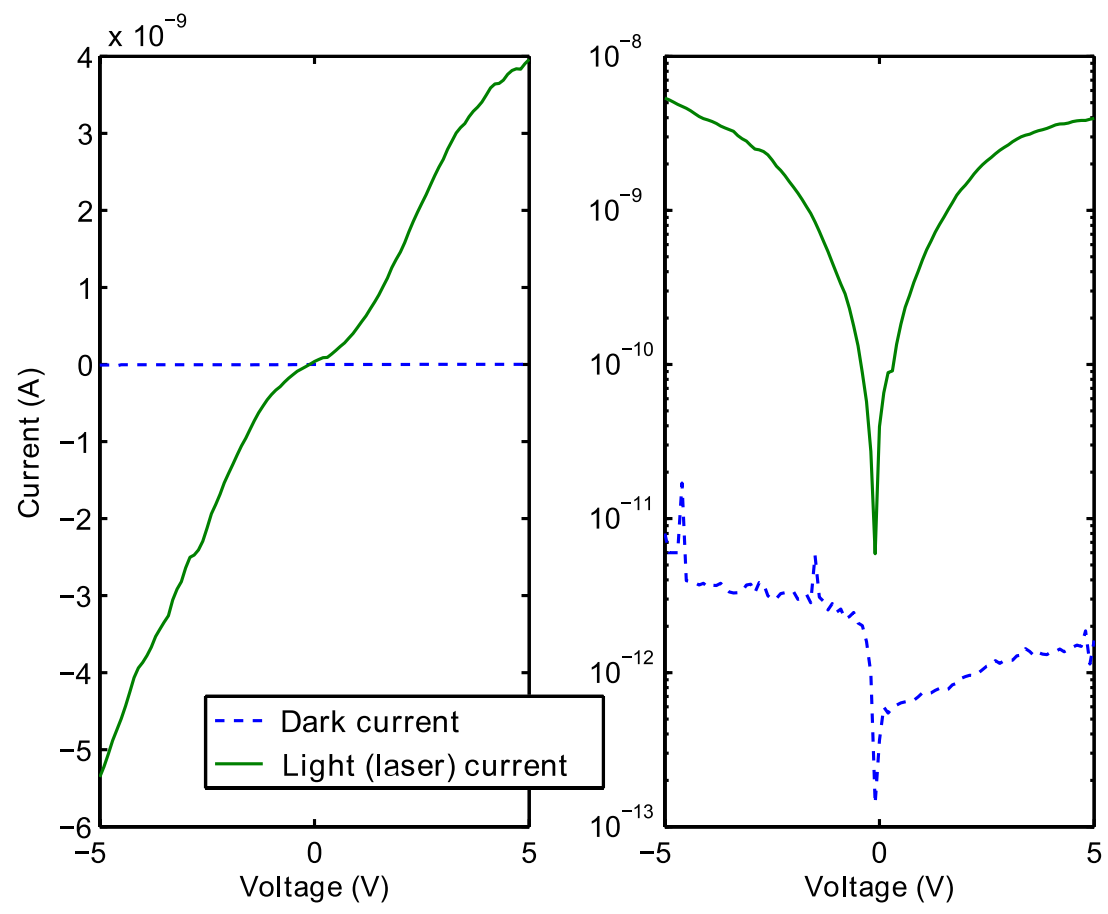

Figure 6. Room-temperature $I V$ measurements on the contacted single GaAs-AlGaAs nanowire shown on linear (left) and logarithmic (right) scales. A peak light to dark current ratio of 3000 is observed. 
photoluminescence, the technique of direct-write lithography represents a positive step towards the larger-scale integration of nanowires into conventional microelectronics.

\section{Acknowledgments}

The authors acknowledge the financial support of the Australian Research Council (Grant: FL0992306). Facilities used in this work are supported by the Australian National Fabrication Facility.

\section{References}

[1] Cho C-H, Aspetti C O, Turk M E, Kikkawa J M, Nam S-W and Agarwal R 2011 Tailoring hot-exciton emission and lifetimes in semiconducting nanowires via whispering-gallery nanocavity plasmons Nature Mater. 10 669-75

[2] Claudon J, Bleuse J, Malik N S, Bazin M, Jaffrennou P, Gregersen N, Sauvan C, Lalanne P and Grard J-M 2010 A highly efficient single-photon source based on a quantum dot in a photonic nanowire Nature Photon. 4 174-7

[3] Scheffler M, Nadj-Perge S, Kouwenhoven L P, Borgstrom M T and Bakkers E P A M 2009 Diameter-dependent conductance of InAs nanowires J. Appl. Phys. 106124303

[4] Chen G, Gallo E M, Burger J, Nabet B, Cola A, Prete P, Lovergine N and Spanier J E 2010 On direct-writing methods for electrically contacting GaAs and ge nanowire devices Appl. Phys. Lett. 96223107

[5] Parkinson P, Lloyd-Hughes J, Gao Q, Hoe Tan H, Jagadish C, Johnston M B and Herz L M 2007 Transient terahertz conductivity of GaAs nanowires Nano Lett. 7 2162-5

[6] Fan Z, Ho J C, Jacobson Z A, Yerushalmi R, Alley R L, Razavi H and Javey A 2008 Wafer-scale assembly of highly ordered semiconductor nanowire arrays by contact printing Nano Lett. 8 20-5
[7] Tomioka K, Kobayashi Y, Motohisa J, Hara S and Fukui T 2009 Selective-area growth of vertically aligned GaAs and GaAs/AlGaAs core-shell nanowires on si(111) substrate Nanotechnology 20145302

[8] Li Q, Koo S-M, Richter C A, Edelstein M D, Bonevich J E, Kopanski J J, Suehle J S and Vogel E M 2007 Precise alignment of single nanowires and fabrication of nanoelectromechanical switch and other test structures IEEE Trans. Nanotechnol. 6 256-62

[9] Peng Y, Luxmoore I, Forster M D, Cullis A G and Inkson B J 2008 Nanomanipulation and electrical behaviour of a single gold nanowire using in situ SEM-FIB-nanomanipulators J. Phys.: Conf. Ser. 126012031

[10] Ibrahim S, Higgins D A and Ito T 2007 Direct-write multiphoton photolithography: a systematic study of the etching behaviors in various commercial polymers Langmuir 23 12406-12

[11] Leon J A, Alves E S, Elias D C, Brant J C, Barbosa T C, Malard L M, Pimenta M A, Ribeiro G M and Plentz F 2011 Rapid fabrication of bilayer graphene devices using direct laser writing photolithography J. Vac. Sci. Technol. B 29021204

[12] Joyce H J et al 2009 Unexpected benefits of rapid growth rate for III-V nanowires Nano Lett. 9 695-701

[13] Jiang N, Parkinson P, Gao Q, Breuer S, Tan H H, Wong-Leung $\mathrm{J}$ and Jagadish C 2012 Long minority carrier lifetime in Au-catalyzed GaAs/AlxGa1xAs core-shell nanowires Appl. Phys. Lett. 101023111

[14] Haske W, Chen V W, Hales J M, Dong W, Barlow S, Marder S R and Perry J W $200765 \mathrm{~nm}$ feature sizes using visible wavelength 3-D multiphoton lithography $O p t$. Express 153426

[15] Thunich S, Prechtel L, Spirkoska D, Abstreiter G, Fontcuberta i Morral A and Holleitner A W 2009 Photocurrent and photoconductance properties of a GaAs nanowire Appl. Phys. Lett. 95083111

[16] Tajik N, Peng Z, Kuyanov P and LaPierre R R 2011 Sulfur passivation and contact methods for GaAs nanowire solar cells Nanotechnology 22225402 